\title{
Cross-national research in audiovisual translation: Some methodological considerations
}

Elisa Perego

University of Trieste

Citation: Perego, E. (2018). Crossnational research in audiovisual translation: Some methodological considerations. Journal of Audiovisual Translation, 1(1), 64-80.

Editor(s): A. Jankowska \& J. Pedersen

Received: January 22, 2018

Accepted: June 30, 2018

Published: November 15, 2018

Funding: University of Trieste Research Fund FRA 2013 and ADLAB PRO project.

Copyright: @2018 Perego. This is an open access article distributed under the terms of the Creative Commons Attribution License. This allows for unrestricted use, distribution, and reproduction in any medium, provided the original author and source are credited.

\section{Abstract}

Cross-national research is a research methodology that aims to make comparisons across countries. Nowadays, cross-national research is growing, especially in some social science disciplines such as sociology, political science, geography, and economics. However, it has not yet steadily entered the field of audiovisual translation (AVT). Despite its theoretical and methodological challenges (Hoffmeyer-Zlotnik \& Harkness, 2005), cross-national research could be used successfully in AVT to investigate long-debated issues. These issues include, but are not restricted to, the reception of a given AVT method or strategy, and the different ways in which the translation process is accomplished in different countries. In this contribution, I will outline both theoretical and methodological debates in comparative cross-national research and apply them to AVT. After that, I will briefly present the results of a cross-national study (Perego et. al, 2016) designed to analyse the subtitle reception comparing the way the process takes place in different countries with different AVT traditions. The discussion of the study will contribute to assessing the potential strengths of cross-national research in AVT, thus offering indications on future methodological developments and enabling future and more rigorous international comparisons.

Key words: cross-national research, EU project, methodology, subtitling, familiarity

凶eperego@units.it; https://orcid.org/0000-0001-6075-4371 


\section{Cross-national research: definition and applications}

Cross-national research is a technique of analysing an event or process that takes place within a country, while comparing the way that event or process takes place across many different countries and societies. In other words, it is a research methodology that aims to compare the manifestation of particular issues or phenomena in different socio-cultural settings - using the same research instruments to seek explanations for similarities and differences - and to gain a greater awareness of a given reality (Hantrais, 1995; Hantrais \& Steen, 2007; Harzing, Reiche \& Pudelko, 2012; Hoffmeyer-Zlotnik \& Harkness, 2005; Jürgen \& Hoffmeyer-Zlotnik, 2003). The methods adopted in cross-national research are no different from those used for domestic (within-nation) approaches. However, cross-national research faces additional methodological complexities which requires special attention to establish equivalence in terms of adopted design, sample, stimuli, and measures (Behr \& Scholz, 2011; Hantrais, 1995; Harzing \& Reiche, 2007).

Nowadays, cross-national research is growing, especially in some social science disciplines such as sociology, political science, geography, and economics. However, it is not yet firmly established in the field of audiovisual translation (AVT). Within-nation approaches to specific translation problems or translation reception questions are still favoured, as demonstrated by empirical studies that have been conducted quite extensively, especially in some countries. Regarding subtitling, mono-national empirical research has been conducted widely, especially in Belgium, Italy, Poland, Spain, Switzerland, and Belgium (cf. the works of Géry d'Ydewalle and his team but also, e.g., Krejtz, Szarkowska, \& Krejtz, 2013; Kruger, Szarkowska, \& Krejtz, 2015; Orrego-Carmona, 2015; Perego, Del Missier, Porta \& Mosconi, 2010; Perego, Del Missier \& Bottiroli, 2015; Perego et al., 2016; Szarkowska, 2010; Wissmath, Weibel, \& Groner, 2009), as well as more recently in the US (Hinkin, Harris, \& Miranda, 2014). Regarding audio description for the blind and visually impaired, mono-national empirical research has been conducted in Poland (Krejtz, Szarkowska, Krejtz, Walczak \& Duchowski, 2012), in Italy (Perego, 2016) and in the UK (Fryer \& Freeman, 2012, 2013, 2014); even the first surveys and empirical studies in this area date back to the early 1990s (Frazier \& Coutinho-Johnson, 1995; Peli, Fine, \& Labianca, 1996; Schmeidler \& Kirchner, 2001).

In fact, collective research initiatives in the field of AVT using a cross-national perspective do exist, and most, if not all, have been implemented in EU projects such as the Pear Tree, Audio Description: Lifelong Access for the Blind (ADLAB, http://www.adlabproject.eu/home/), Digital Television for All (DTV4ALL), Hybrid Broadcast Broadband for All (HBB4ALL, http://pagines.uab.cat/hbb4all/), Accessible Culture and Training (ACT, http://pagines.uab.cat/act/), Audio Description: A Laboratory for the Development of a New Professional Profile (ADLAB PRO, https://www.adlabpro.eu/) and Interlingual Live Subtitling for Access (ILSA, http://www.ilsaproject.eu/) projects. In the studies conducted as part of the project activities, participants from different European countries were asked to perform the same activity with data then subjected to comparative analyses to uncover similarities 
and differences in the way certain tasks were performed by representatives of the languages and cultures concerned.

Taylor \& Mauro's (2012) Pear Tree project was carried out internationally at 11 universities in Europe (France, Germany, Great Britain, Greece, Ireland, Italy, Belgium, Poland, and Spain), the United States, and the Republic of South Africa (involving Afrikaans-speaking participants). Based on a research vehicle devised by Chafe (1980), participants were shown a short video clip without dialogue and were asked to write down what they had seen. The project was designed with the aim of enabling an intercultural comparison of the film descriptions in order to study verbalisation modalities across languages and cultures (Gronek, Gorius \& Gerzymisch-Arbogast, 2012; Mazur \& Kruger, 2012; Taylor \& Mauro, 2012). The DTV4ALL project was a European venture designed to facilitate the provision of access services on digital television across the European Union. Focusing, among other things, on subtitling for the deaf and hard of hearing (SDH), it produced the first volume to deal specifically with the quality of SDH in Europe, with contributions from the UK, Spain, Italy, Poland, Denmark, France and Germany (Romero-Fresco, 2015). In the volume, several aspects of the reception of SDH involve populations living in different countries. The HBB4ALL project included 12 partners from several complementary fields (universities, TV channels and broadcasters, research institutes, and small and medium enterprises) that were experts in media accessibility. Through testing in different countries and EU languages, the project examined the reactions to the delivery of TV content via PC, tablet, smartphone and TV with an array of communication solutions such as subtitling, audio description, clean audio, and many customizable features. The $A D L A B$ project was designed to create effective and reliable guidelines for the practice of audio description that could be usable throughout Europe. Best practices and recommendations were established through research and field work, while also relying on testing with blind end-users. Testing was carried out in six European countries (including Belgium, Germany, Italy, Poland, Portugal and Spain) using a carefully prepared questionnaire to be completed after the viewing of different AD versions of a vehicle film, Inglourious Basterds (Bender \& Tarantino, 2009), chosen because of its complex content and narrative. The questionnaire matrix in English was annotated and translated to ascertain the levels of comprehension and enjoyment of different types of audio description, and were then presented to linguistically and culturally diverse blind audiences (ADLAB, 2013; Maszerowska, Matamala, \& Orero, 2014). Based on the efficacy of the methodology employed by replicating the partnership, core activities and experimental design, currently funded AVT projects in the EU have used an empirical approach encompassing a cross-national perspective which have benefitted from comparative research; three relevant instances are represented by ACT, ADLAB PRO and ILSA. All projects are financed by the European Union under the Erasmus+ Programme, Key Action 2 - Strategic Partnerships, and besides methodology, they share a strong focus on media accessibility in diverse forms. The ACT project aims to promote equal opportunities and accessibility to cultural events across countries. It also proposes the definition of a new professional profile of Media Accessibility Expert/Manager for the Scenic Arts, as well as various types of associated training activities (Orero, 2017). The ADLAB PRO (Audio Description: A Laboratory for the Development of a New Professional Profile) project will develop and define a curriculum 
for ensuring professional Europe-wide $A D$ implementation in all cultural and media sectors through the creation of training materials (Perego, 2017). The ILSA project aims to design, develop, test and recognise the first training course for interlingual subtitling for the deaf and hard of hearing, and to produce a protocol for the implementation of this service in three real-life scenarios (such as TV, classroom and Parliament).

Testing or surveying in all partner countries is a key defining step of each project because it can rely on cross-national data, offer a broad and comprehensive perspective on several issues, and compare the stance of respondents within a different cultural and linguistic background. A whole intellectual output (IO) - an EU-specific expression referring to a working activity to be accomplished over a precise timeframe and leading to tangible results - of ADLAB PRO was based on comparable feedback gained by respondents from 26 countries (ADLAB PRO, 2017b). 102 focused on setting out a list of skills and competencies needed to train audio description professionals to produce the necessary data for project partners who are in charge of designing a course curriculum and producing training materials to form the figure of the audio describer. Given the international nature of this professional figure, it was important to gain as wide a perspective as possible and compare responses that came from different realities.

All projects (ACT, ADLAB PRO and ILSA) included an initial phase based on the collection of international and cross-national data through survey questionnaire in order to investigate current training practices in terms of accessibility, audio description and interlingual live subtitling respectively, as well as offer a snapshot of the situation in Europe. The survey results represented an invaluable starting point "for obtaining a clear understanding of what is common to all training programmes and what is still required in the provision of a professional curriculum" (ADLAB PRO, 2017a, p. 2).

\section{Merits and challenges of cross-national research}

These instances of comparative research in the field of AVT bring us to the various merits of crossnational research. These include the possibility of uncovering similarities and differences when studying the same phenomenon; to come to a deeper understanding of a given product or process; to replicate experiments in countries with the desirable effect of working with greater or different population samples and therefore to generalize results; to use the same research toolkit in different countries; to strengthen research networks or to create new ones; and finally, to establish more comprehensive and coherent theoretical frameworks that explain how specific sets of phenomena work. These are all invaluable assets in the field of AVT, which is still working to find its way methodologically (Orero et al., 2018). A cross-national experimental design could contribute to overcoming several weaknesses or inconsistencies which have long characterized and hindered AVT research, such as recruitment difficulties, particularly with vulnerable users. 
The main strength of the ADLAB report on testing (ADLAB, 2013) was that it involved a large group of respondents ( 80 people with visual impairment and 77 sighted controls), a result of the collaboration and strong commitment from the research team. This is particularly relevant because it is rarely feasible to reach such a large group of participants in AVT reception studies, especially when vulnerable audiences are involved. In the case of the ADLAB study, a large sample of respondents guaranteed that the preferences of all groups of people with visual impairment could be considered and compared in order to gain deeper understanding of their attitude towards $A D$ in different national contexts with different AVT traditions. Choosing to set up a cross-national experiment or project is also beneficial in terms of partnership. In cross-national research projects, it is crucial to choose a consortium based on a diversification principle, which allows a blend of complementary organizations that combine several academic disciplines and perspectives into one activity. By drawing on a highly diversified combination of expertise, these projects also provide an example of real cooperation and networking, thus merging the traditional research boundaries in line with the principles of interdisciplinarity. Furthermore, the languages and cultures involved can be numerous, to include both widely spoken and lesser-used languages from diverse families, covering a diversified array of cultural environments and traditions.

Overall, in the field of AVT, cross-national research is particularly important as its results could lead to the creation of flexible and shareable guidelines, instead of rigid and prescriptive ones, for each AVT method. As a result, AVT practices across Europe could be harmonized, and the training and practice of AVT professionals could be based on a shared set of best practices and the real needs and preferences of end-users. This was made clear in the scope of both the ADLAB and the DTV4ALL projects. The cross-national studies conducted so far, however, seem to confirm that the audiovisual landscape and viewers' preferences are very heterogeneous and that overcoming the existing local norms is not easy nor necessary, given the current practice of many service providers that outsource translations to a single company who use similar standards for various markets. In fact, a common core and some shared approaches still exist along with national specificities, which need to be respected (Romero-Fresco, 2015).

However, cross-national research can also pose some challenges. From a practical perspective, it is time-consuming and based on teamwork and collaboration, which falls outside the tradition of researchers in the field of humanities. From a methodological perspective, researchers are typically worried about the translation of research material; the comparability, reliability and validity of data; the harmonization of data and experimental procedures; and the differences in research traditions and cultural interference (Behr \& Scholz, 2011; Hoffmeyer-Zlotnik \& Harkness, 2005; Hantrais, 1995; Harzing, 2007). The ADLAB (2013) report on testing exemplifies some of the major challenges that cross-national research in AVT can encounter. In particular, the authors of the report stress the fact that testing was done in the local languages of the countries where the tests were administered. Consequently, respondents were exposed to a translated version of the stimuli originally prepared in English (used as a pivot language, since the original ADs were developed in German and Dutch) and responded to questionnaires translated into local languages (Dutch, 
German, Italian, Polish, Portuguese, Spanish) from English. As we will emphasise in the following sections of this paper, the translation of questionnaires is a central issue in cross-national research where "both a high-quality source questionnaire and high-quality translations are central to the overall comparability of survey data" (Behr \& Scholz, 2011, p. 158). This was why the translation of the questionnaires was carried out collaboratively - to ensure that "the translated items measure what they [were] supposed to measure and that the equivalence to the source questionnaire [was] not lost because of a flawed translation" (Behr \& Scholz, 2011, p. 159).

More applied instances of cross-national research challenges will be presented in the next section, where further methodological considerations will stem from the description of a case study specifically conducted on the reception of subtitling.

\section{A cross-national study on the reception of subtitling}

Cross-national research was the guiding principle of the University of Trieste Research Fund FRA 2013 entitled, Towards an empirical evaluation of audiovisual translation: A new integrated approach. The project aimed to study audiovisual translation from a receptive perspective and used a new field approach to identify the real and supposed advantages and disadvantages of specific forms of AVT. It combined theoretical and methodological contributions from different disciplines such as translation, linguistics and psychology (Perego, 2014). The University of Trieste was the coordinating partner of the project, which also included a network of partners from the Universities of Antwerp, Barcelona, Pavia and Warsaw.

Aware of the merits and the challenges of cross-national research, we set up a cross-national study on subtitle reception in order to assess whether cross-national commonalities or differences existed in the way subtitled products were used, processed and enjoyed, and in the way they shaped the preferences and future choices of users, depending on the familiarity they had with subtitles (Perego et al., 2016). The study was carried out in four European countries (Italy, Spain, Poland and Belgium) with different AVT traditions (dubbing, subtitling and voice-over) that were representative of different distribution and familiarity patterns for AVT. According to the literature, Italy and Spain were dubbing countries, Poland was a voice-over country and Belgium was a subtitling country (Danan, 1991; Luyken, Herbst, Langham-Brown, Reid \& Spinhof, 1991). This, however, did not seem to reflect accurately the current situation described in more recent sources (Chaume, 2012; Kuipers, 2015; Media Consulting Group, 2007, 2011; Szarkowska \& Laskowska, 2015) and was only partially reflected in the viewing habits of the users participating in the study. As shown in Figure 1 , participants were asked to mark on a 7-point Likert scale how frequently they used subtitles to access foreign AV material. Belgians and Poles were the more frequent subtitle users, especially if compared to the Italian group of participants, who only used subtitles occasionally. This latter figure confirmed that dubbing was not the only AVT method accessed by the Italian population, even in the recent past. Both recent literature and the answers from the experiment indicated that participants seemed 
to confirm a slow and steady shift of usage and preference towards subtitling being experienced in countries where other AVT methods were originally favoured. This shift did not change the situation dramatically; Italy and Spain still used dubbing and Poland still used voice-over, whereas Belgium always only used subtitling. However, it suggested that it was no longer possible to talk about viewers that were completely unfamiliar with subtitles.

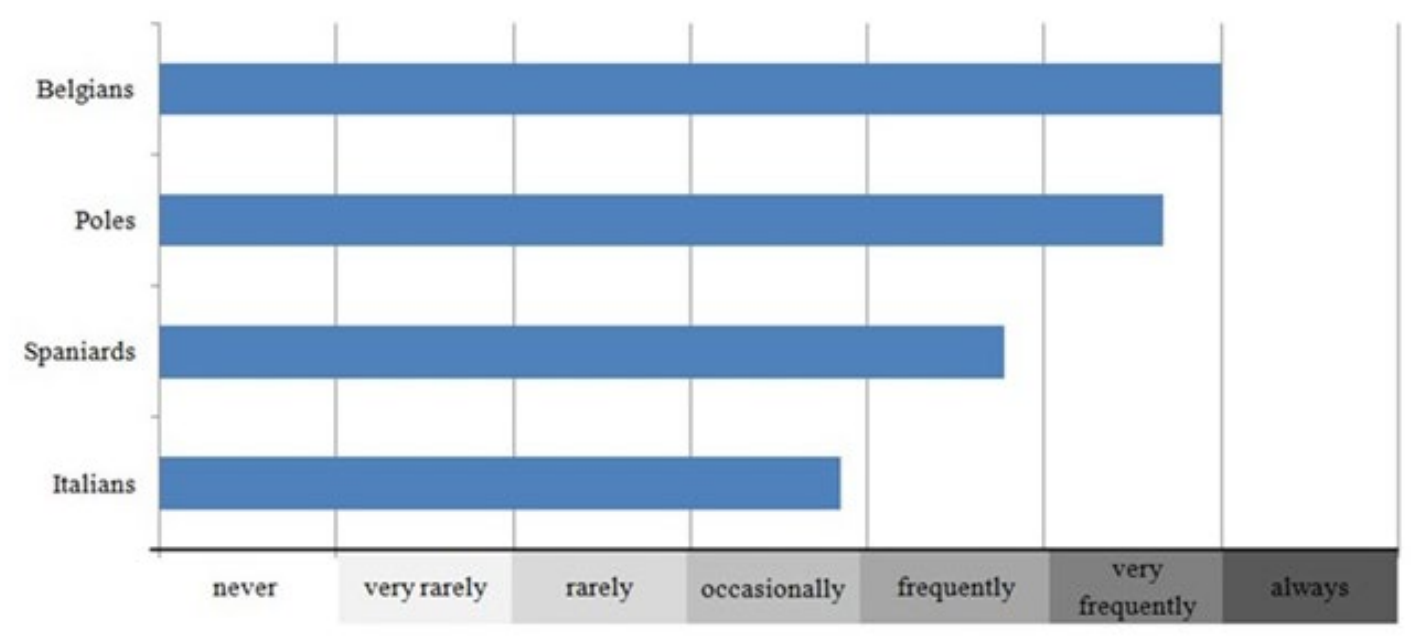

Figure 1. Frequency of subtitle use declared by the participants (Perego et al., 2016).

For different reasons, ranging from cultural to economical, subtitles had taken over across all European countries thanks to new technologies and devices such as smartphones and smart TVs, thus setting aside the long-standing popularity of other forms of AVT (O'Sullivan, 2015).

\subsection{Materials and methodology}

The study involved 113 participants aged in their 20s. Participants were undergraduate and postgraduate students of translation and interpreting, applied linguistics and psychology: 34 Italians (26 females, 19-25 years); 26 Spaniards (19 females, 20-30 years); 20 Poles (14 females, 18-29 years); and 33 Belgians (25 females, 19-26 years). The response rate differed slightly across countries, despite the study's attempt to maximise it, which reflected a typical challenge of crossnational research (Harzing et al., 2012). Incentives such as ECTS and financial tokens were not used to increase response rate. Low response rate often represents an issue in experimental research, even more so in the field of AVT (Orero et al., 2018). The final number of respondents was also impacted by different university selection and recruitment policies, such as restrictions on approaching students and difficulty in recruiting participants in other departments. A careful evaluation of the recruitment principles and procedures of all institutions involved, as well as possible local constraints, are therefore crucial to ensure qualitatively and quantitatively comparable samples, and consequently, comparable results in all countries involved. Setting up a shared document on subject selection, recruitment and compensation would enable the research team to follow consistent protocols from the onset of the research, and when identifying participants 
to join the study. In the field of AVT, however, established practices are in the making and still need to be fine-tuned in this respect (Orero et al., 2018).

The film used in the experiment was the critically-acclaimed Caramel (Toussaint \& Labaki, 2007), and described as "both an astute cultural study, and a charming comedic drama" (Rotten Tomatoes, 2008). Set in Beirut, the film was equally distant in a cultural point of view from all the countries involved in the research. The plot was not heavily marked, and it tackled themes that could still be taboo in Muslim culture, such as pre-marital sex, adultery, unaccepted ageism and homosexuality. During the experiment, participants watched the 25-minute opening scene of the film in Lebanese Arabic (an unknown language to all participants) with professional DVD subtitles in their mother tongue, and then answered questions assessing their comprehension of the film plot. These questions tested the following: their memory for words used in the subtitles and images they were exposed to; their appreciation of the film viewing experience; their viewing habits; and their future intentions regarding the film. This was measured by replies to these questions: would they watch the whole film; watch another film by the same director or same actors; or would recommend the film to a friend. They were also administered cognitive tests: Raven's Coloured Progressive Matrices as a measure of fluid intelligence; Letter and Pattern Comparison Tasks as indicators of processing speed; and a Vocabulary Test as a measure of verbal ability (Perego et al., 2015; Perego et al., 2016). Watching the excerpt and answering the questionnaire took approximately 60 minutes.

The use of cognitive tests added interesting insight to a reception experiment, because it assessed participants' individual differences and correlated them with the results. However, since some of these tests could only be administered by professional psychologists, the appropriate way to proceed needs to be found. Research teams from different countries should each include a psychologist to communicate and negotiate with the psychologists from the other teams in order to harmonize the test administration procedures, the analysis of the data, and, in some cases, decide on the selection of tests to be used. Not all tests are available in all languages, and it might be important to find those which can be easily used and compared in the countries involved in the experiment.

Setting up the cross-national study involved the preparation of a questionnaire template with annotations in English (Behr \& Scholz, 2011) as seen in Example 1. The questionnaire was translated into the local languages of the countries involved in the experiment. The annotations were meant to guide the translator in the choice of translation procedures (direct vs. oblique) or to disambiguate the wording of the source text when necessary. They aimed at a target text that could be as close as possible to the source text in terms of conceptual equivalence and vocabulary choices. 


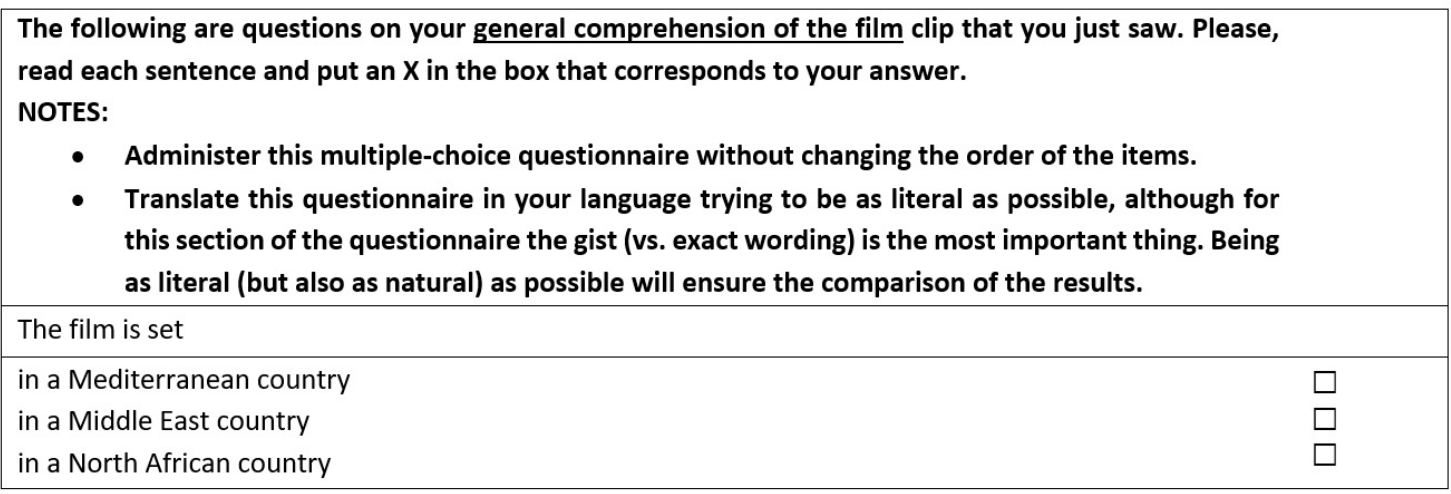

Example 1. Excerpt of the annotated questionnaire matrix

The choice to translate the questionnaire into the native language of the respondents was based on research findings showing that in cross-national research, the use of English-language questionnaires might create a language bias (Harzing et al., 2012). As the authors illustrate clearly:

Further research even showed that the language of the questionnaire can impact not just attitudes, but also behaviours (Akkermans, Harzing \& van Witteloostuijn, 2010). Especially if both native and non-native English speakers are included in an international survey, survey translation into the respective local language appears crucial. Finally, translation might also have a symbolic effect. Even if respondents are comfortable with English, translation demonstrates to the respondent that the researcher has gone through the effort and expense to make responding as easy as possible. This might well influence response rates (Harzing et al., 2012, p. 18).

When necessary, the questionnaire was also adapted to suit different language subtitles as shown in Example 2. Compared to the original dialogues, the length and completeness of the interlingual subtitles differed depending on the language involved. In the cases illustrated below, Italian subtitles tended to reproduce more linguistic material whereas Spanish subtitles were more concise and compressed or omitted portions of text. This translation behaviour had to be taken into account when translating the questionnaire, which in some cases needed specific adaptations. 


\begin{tabular}{|c|c|}
\hline Spanish subtitles & Italian subtitles \\
\hline $\begin{array}{l}\text { 00:01:38,520 -> 00:01:40,681 } \\
\text { iListo! } \\
\text { (Ready!) } \\
\text { 00:01:55,336 -> 00:01:57,201 } \\
\text { Espero poder } \\
\text { arreglarte para tu boda. } \\
\text { (I hope I can } \\
\text { arrange for your wedding). } \\
\text { 00:01:57,338 -> 00:01:59,306 } \\
\text { iTú primero! } \\
\text { (You first!) } \\
\text { 00:02:22,163 -> 00:02:24,222 } \\
\text { Layale, escucha carińo. } \\
\text { (Layale, listen honey.) } \\
\text { 00:02:24,365 -> 00:02:27,027 } \\
\text { Tengo una audición para un anuncio } \\
\text { importante y voy tarde. } \\
\text { (I have an audition for an important } \\
\text { ad and I'm late.) } \\
\text { 00:02:27,168 -> 00:02:29,534 } \\
\text { Por favor, atiéndeme } \\
\text { antes que a la pelirroja. } \\
\text { (Please, help me } \\
\text { with the redhead.) }\end{array}$ & $\begin{array}{l}\text { 00:01:51,687 --> 00:01:53,279 } \\
\text { Un momento. } \\
\text { (Just a moment.) } \\
\text { 00:02:02,527 --> 00:02:06,520 } \\
\text { - Voglio essere io a farti bella } \\
\text { quando ti sposerai. - Dopo di te. } \\
\text { (- I want to be the one to make you look nice } \\
\text { when you get married. - After you.) }\end{array}$ \\
\hline
\end{tabular}

Example 2. Different wording in the Spanish and Italian subtitles of the initial scenes of Caramel (Toussaint \& Labaki, 2007). A back translation is offered in brackets, under the subtitles.

Experimental sessions took place locally in each country after a recruitment period and pilot testing. Questionnaires were administered on paper - although a convenient alternative in a cross-national setting, especially with long questionnaires and a large sample of participant per country, would be online distribution. Online distribution would also enable researchers to address questionnaire accessibility issues and distribute accessible questionnaires to people with sight loss (ADLAB PRO 2017b). Finally, data was gathered, collated and analysed statistically using the SPSS Statistics software package.

From the methodological point of view, it was crucial to tackle and homogenise the ethics procedures, recruit comparable samples of respondents (which proved to be challenging), and make sure that the cognitive tests (Raven's CPM; Letter and Pattern Comparison Tasks; Vocabulary Test) were established and recognized in all the countries involved. 
A data entry template with instructions was created and shared as well as a detailed codebook (or data dictionary) telling the coder how each questionnaire was coded for data entry (Example 3). This ensured that data was entered in the spreadsheet the same way by all coders.

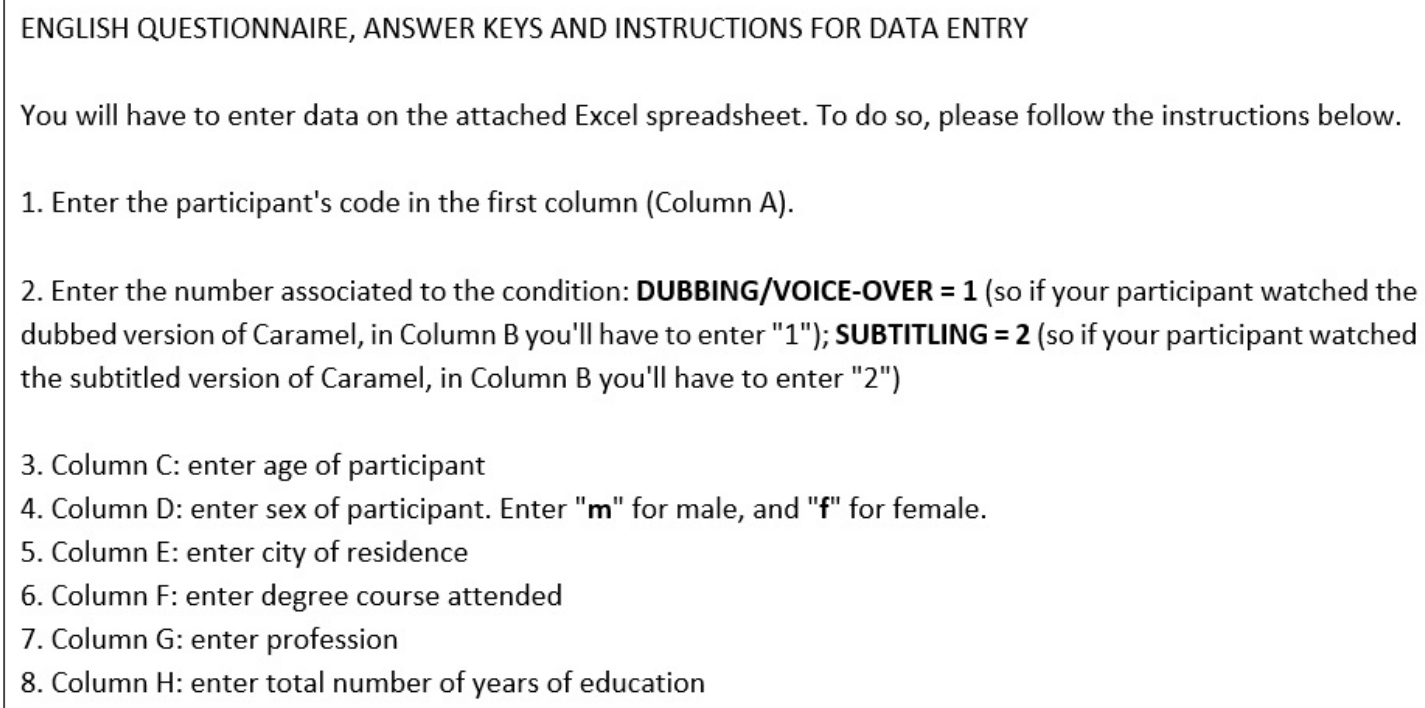

Example 3. Excerpt of the answer keys and data entry instructions.

A further methodological issue to be tackled was the use of specific research methods such as eyetracking, electroencephalography, psychometrics and electro dermal activity, and heart rate. These methods are being used more and more often in AVT empirical research to produce complementary measures that prove to be very useful in the investigation of the processing of AVT products. In fact, these informative online measures allow the researcher to collect data while the participant processes the text, which can complement the more traditional post-hoc offline measures used directly after participants have been exposed to a text, such as self-reported cognitive effort scales, presence or transportation scales, comprehension or retention questionnaires, narrative reports, interviews or focus groups (Orero et al., 2018). In a cross-national context, the decision to use these tools implied the need to include experts, use the same or comparable equipment, or produce the same sets of data in order for them to be comparable. In the case of this study, eye-tracking sessions were carried out in two of the four countries involved due to the lack of the necessary equipment. For this reason, eye tracking data was not included in the final data set but was used independently by researchers.

To conclude, although data was collected internationally, the project was centrally controlled and most of the design was executed by only one researcher.

In this section of the paper, we will provide a brief overview of the results of the study we have been examining (Perego et al., 2016) and discuss a series of methodological issues related to cross-national research in AVT. Since the study itself and its results are not the focus of this paper, we are not including details and quantitative results. However, the major findings and observations 
are reported below for the sake of completeness. Overall, results showed that familiarity could influence at least some aspects of the film viewing experience. In particular, they showed that subtitling was cognitively effective irrespective of familiarity. In fact, this AVT method enabled full understanding of the film plot; it facilitated memory for exact wording, and was not perceived as taxing or challenging but as helpful and easy to follow. However, in spite of its cognitive effectiveness, subtitling was not equally effective from an evaluative point of view - those who were less familiar with subtitles enjoyed the film experience less, appreciated dialogues less, and appreciated the original (unknown) voices of the actors less. Despite a lessened appreciation of the subtitled film, the results had interesting implications; they suggested that subtitling could be exploited more generally than it is today to enhance literacy and language acquisition (Kothari, 2000; Vanderplank, 2016), as well as to increase familiarity with subtitling itself. This is in fact a prerequisite for appreciating it more and enjoying its language-related benefits. Regarding viewers' future intentions, results also showed that these seemed to be shaped by their having been exposed to subtitling. When confronted with the following questionnaire item:

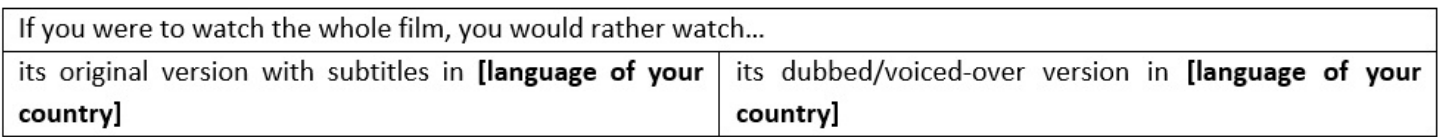

Example 4. Excerpt of the questionnaire.

27 Italians (vs. 8), 20 Spaniards (vs. 5), and 17 Poles (vs. 3) claimed that they would watch the whole film with subtitles if they could choose. This suggested that familiarity patterns could be easier to change than expected. In particular, we believe that this finding could be exploited, especially in dubbing countries, to the advantage of a series of potential beneficiaries of subtitles. This includes motivated language learners, illiterate users, immigrants that must learn the language of their host country, but also elderly people with hearing loss that find themselves having to turn from dubbed to subtitles products to access AV material.

Another interesting finding was that, contrary to earlier findings (Perego et al., 2010; Perego et al., 2015), subtitling seemed to interfere with the full processing of the visual track. This seemed to indicate that the eyes of the viewers spent more time reading subtitles (Marleau, 1982) or that subtitle processing may require some effort after all, especially for those who are not habitual subtitle users. In particular, Italians - who claimed that they use subtitles occasionally (Figure 1) - expectedly showed the worst performance in visual scene recognition. However, and quite unexpectedly, the Poles showed a poor performance despite their familiarity with subtitling. They claimed that they use subtitles very frequently, and this was also confirmed in literature, which considers Poland a mixed country and not a stronghold of voice-over any longer (Szarkowska \& Laskowska, 2015). The reason for the poor performance of the Poles might lie in some specific methodological limitations of the study, including the fact that the sample of Polish participants was smaller than the other samples; that the Polish subtitles were longer than subtitles in other languages in terms of average number of characters per line; and that Polish subtitles 
were more numerous. These limitations, however, could easily be prevented, especially in largerscale projects. Larger-scale projects would have more resources to cope with the need to manage different language stimuli and questionnaires, be able to coordinate with country collaborators, secure an appropriate sample of participants, and comply with the data collection process that requires substantially more time than domestic research (Hantrais, 1995; Harzig, 2007). Although we opted for the use of original material, we agreed that for the sake of results, it could be useful to construct comparable stimuli and recruit larger and more homogeneous groups of participants. There still needs to be full awareness of new methodological approaches in AVT to fix the current flaws and offer further exciting directions and solutions for cross-national research.

\section{Concluding remarks}

After defining cross-national research, this paper briefly illustrated the few, yet seminal, crossnational research initiatives that have been carried out in the field of AVT (the Pear Tree project, the ADLAB project, the DTV4ALL project, the HBB4ALL project, and more recently the ACT, ADLAB PRO and ILSA projects) and reported on the results of a reception study on subtitling that was conducted in four European countries. In doing so, it shed light on the advantages, as well as the additional requirements and complexities of cross-national research in the field of AVT, where this approach was not yet established and full awareness of its methodological aspects should be developed.

The AVT field is still flexible and trying to set an established research framework (Orero et al., 2018). Within this new, more methodologically rigorous framework, AVT could benefit greatly from a more aware and systematic cross-national approach to some of its core issues, especially those linked to vulnerable audience reception. Knowing the preferences and the reactions of larger groups of audiences from different EU (and non-EU) countries to given AVT methods, this could be exploited to maximize their quality, usability, appreciation, and distribution policies. Testing specific solutions for audiences with different linguistic and cultural backgrounds would help identify universal and language specific AVT strategy effectiveness and how these can be used to benefit the audience. The results of AVT studies that adopted this perspective have been promising and show that cross-national research is a great opportunity also in this field. AVT could benefit considerably from the development of this approach, especially due to the strong encouragement towards the interdisciplinary and international collaboration and networking required. The nature of most European project funding initiatives seem to encourage international comparisons as a way forward. Strengthening and expanding the methodological awareness of AVT will give it the deserved space in a wider EU framework and allow it to contribute concretely to the development of a more comprehensive and competitive research. 


\section{Acknowledgements}

Preparation of this article was supported by the University of Trieste Research Fund FRA 2013 (Towards an empirical evaluation of audiovisual translation: A new integrated approach) and by the ADLAB PRO (Audio Description: A Laboratory for the Development of a New Professional Profile) project, financed by the European Union under the Erasmus+ Programme, Key Action 2 Strategic Partnerships, Project number:2016-1-IT02-KA203-024311, both awarded to Elisa Perego.

The information and views set out in this publication are those of the author and do not necessarily reflect the official opinion of the European Union. Neither the European Union institutions and bodies nor any person acting on their behalf may be held responsible for the use which may be made of the information contained therein.

\section{Biographical note}

Elisa Perego, PhD, is Associate Professor of English Language and Linguistics at the University of Trieste, Italy, where she teaches English linguistics and translation, translation theory, and research methodology in translation studies. Her research interests and publications mainly focus on AVT accessibility and reception; subtitling and audio description, which are studied mainly from a cognitive perspective; empirical and cross-national research methodology. Elisa is currently the coordinator of the European project ADLAB PRO (2016-2019) on audio description, and she is a partner in the European project EASIT (2018-2021) on easy to understand language in audiovisual translation.

\section{References}

ADLAB. (2013). Course design. Project report. Retrieved from https://www.adlabpro.eu/wpcontent/uploads/2018/11/ADLABPRO IO3 Report.pdf

ADLAB PRO. (2017a). Assessment of current AD training practices. Retrieved from http://www.adlabpro.eu/wpcontent/uploads/2017/12/20170608 uam io1 report.pdf

ADLAB PRO. (2017b). Audio Description Professional - Profile Definition. Retrieved from https://www.adlabpro.eu/wp-content/uploads/2018/04/IO2-REPORT-Final.pdf

Akkermans, D., Harzing, A.W., \& Witteloostuijn, A. (2010). Cultural accommodation and language priming: Competitive versus cooperative behavior in a prisoner dilemma game. Management International Review, 50(5), 559-584.

Behr, D., \& Scholz, E. (2011). Questionnaire translation in cross-national survey research. Methoden - Daten - Analysen, 5(2), 157-179.

Bender, L. (Producer), \& Tarantino, Q. (Director). (2009). Inglorious Basterds [Motion picture]. United States: The Weinstain Company \& Universal Pictures. 
Chafe, W. (1980). The Pear Stories: Cognitive, cultural and linguistic aspects of narrative production. Norwood, NJ: Ablex.

Chaume, F. (2012). Audiovisual translation: Dubbing. Manchester: St. Jerome.

Danan, M. (1991). Dubbing as an expression of nationalism. Meta: Journal des Traducteurs, 36(4), 606-614.

Frazier, G., \& Coutinho-Johnson, I. (1995). The effectiveness of audio description in providing access to educational AV media for blind and visually impaired students in high school. San Francisco: AudioVision.

Fryer, L., \& Freeman, J. (2012). Presence in those with and without sight: Audio description and its potential for virtual reality applications. Journal of Cyber Therapy and Rehabilitation, 5(1), 15-23.

Fryer, L., \& Freeman, J. (2013). Cinematic language and the description of film: Keeping AD users in the frame. Perspectives: Studies in Translatology, 21(3), 412-426.

Fryer, L., \& Freeman, J. (2014). Can you feel what I'm saying? The impact of verbal information on emotion elicitation and presence in people with a visual impairment. In A. Felnhofer \& O.D. Kothgassner (Eds.), Challenging Presence: Proceedings of the 15th International Conference on Presence (pp. 99-107). Wiena: facultas.wuv.

Gronek, A. M., Gorius, A., \& Gerzymisch-Arbogast, H. (2012). Culture and coherence in the Pear Tree Project. Perspectives: Studies in Translatology, 20(1), 43-53.

Hantrais, L. (1995). Comparative research methods. Social Research Update, 13, 1-6.

Hantrais, L., \& Steen, M. (Eds.). (2007). Cross-national research methodology and practice. London: Routledge.

Harzing, A.W., \& Reiche, S. (2007). Key issues in international survey research. Retrieved from https://harzing.com/

Harzing, A.W., Reiche, S., \& Pudelko, M. (2012). Challenges in international survey research: A review with illustrations and suggested solutions for best practice. European Journal of International Management, 7(1), 112-134.

Hinkin, M.P., Harris, R.J., \& Miranda, A.T. (2014). Verbal redundancy aids memory for filmed entertainment dialogue. The Journal of Psychology, 148(2), 161-176.

Hoffmeyer-Zlotnik, J. H. P., \& Harkness, J. A. (2005). Methodological aspects in cross-national research. Mannheim: ZUMA-Nachrichten.

Jürgen, H.P., \& Hoffmeyer-Zlotnik, C. W. (Eds.). (2003). Advances in cross-national comparison: A European working book for demographic and socio-economic variables. New York: Kluwer Academic/Plenum Publishers.

Kothari, B. (2000). Same language subtitling on Indian television: Harnessing the power of popular culture for literacy. In K. Wilkins (Ed.), Redeveloping communication for social change: Theory, practice and power (pp. 135-146). New York: Rowman \& Littlefield.

Krejtz, I., Szarkowska, A., Krejtz, K., Walczak, A., \& Duchowski, A. (Eds.). (2012). Proceedings from ETRA '12: Symposium on Eye Tracking Research and Applications. New York: ACM.

Krejtz, I., Szarkowska, A., \& Krejtz, K. (2013). The effects of shot changes on eye movements in subtitling. Journal of Eye Movement Research, 6(5), 1-12. 
Kruger, J.-L., Szarkowska, A., \& Krejtz, I. (2015). Subtitles on the moving image: An overview of eye tracking studies. Refractory: A Journal of Entertainment Media, 25. Retrieved from http://refractory.unimelb.edu.au/2015/02/07/ kruger-szarkowska-krejtz/

Kuipers, G. (2015). How national institutions mediate the global: Screen translation, institutional interdependencies and the production of national difference in four European countries. American Sociological Review, 80(5), 985-1013.

Luyken, G.-M., Herbst, T., Langham-Brown, J., Reid, H., \& Spinhof, H. (1991). Overcoming language barriers in television. Dubbing and subtitling for the European audience. Manchester, UK: The European Institute for the Media.

Marleau, L. (1982). Les sous-titres... un mal nécessaire. Meta: Journal des Traducteurs, 27(3), 271-28.

Maszerowska, A., Matamala, A., \& Orero, P. (Eds.). (2014). Audio description: New perspectives illustrated. Amsterdam: Benjamins.

Mazur, I., \& Kruger, J.-L. (2012). Pear stories and audio description: Language, perception and cognition across cultures. Perspectives: Studies in Translatology, 20(1), 1-3.

Media Consulting Group. (2007). Study on dubbing and subtitling needs and practices in the European audiovisual industry. European Commission: Directorate-General Education and Culture.

Media Consulting Group. (2011). Study on the use of subtitling: The potential of subtitling to encourage foreign language learning and improve the mastery of foreign languages. European Commission: Directorate-General Education and Culture.

Orero, P. (2017). The professional profile of the expert in media accessibility for the scenic arts. International Journal of Translation, 19, 143-161.

Orero, P., Doherty, S., Kruger, J.-L., Matamala, A., Pedersen, J., Perego, E., Romero-Fresco, P., RoviraEsteva, S., Soler-Vilageliu, O., \& Szarkowska, A. (2018). Conducting experimental research in audiovisual translation: A position paper. The Journal of Specialised Translation, 30, 105126.

Orrego-Carmona D. (2015). The reception of (non) professional subtitling. (Unpublished doctoral dissertation). Tarragona, Universitat Rovira i Virgili.

O'Sullivan, F. (2015, December 7). Spain wants to end TV dubbing to improve English proficiency. CityLab. Retrieved from https://www.citylab.com/life/2015/12/spain-wants-to-end-tvdubbing-to-improve-english-proficiency/419076/

Peli, E., Fine, E., \& Labianca, A. (1996). Evaluating visual information provided by audio description. Journal of Visual Impairment and Blindness, 90(5), 378-385.

Perego, E. (2014). Un nuovo approccio integrato per la valutazione empirica della traduzione audiovisiva. Rivista Internazionale di Tecnica della Traduzione, 16, 189-206.

Perego, E. (2016). Gains and losses of audio description in sighted viewers. Target, 28(3), 424-444.

Perego, E. (2017). Audio description: A laboratory for the development of a new professional profile. International Journal of Translation, 19, 131-142.

Perego, E., Del Missier, F., \& Bottiroli, S. (2015). Dubbing versus subtitling in young and older adults: Cognitive and evaluative aspects. Perspectives: Studies in Translatology, 23(1), 1-21.

Perego, E., Del Missier, F., Porta, M., \& Mosconi, M. (2010). The cognitive effectiveness of subtitle processing. Media Psychology, 13, 243-272. 
Perego, E., Laskowska, M., Matamala, A., Remael, A., Robert I. S., Szarkowska A., Vilaró A., \& Bottiroli S. (2016). Is subtitling equally effective everywhere? A first cross-national study on the reception of interlingually subtitled messages. Empirical Approaches to Audiovisual Translation: Special issue of Across Languages and Cultures, 17(2), 205-229.

Romero-Fresco, P. (2015). The reception of subtitles for the deaf and hard of hearing in Europe. Bern: Peter Lang.

Rotten Tomatoes. (2008). Caramel: Movie reviews, trailers, pictures. Retrieved from http://www.rottentomatoes.com/m/caramel/

Schmeidler, E., \& Kirchner, C. (2001). Adding audio description: Does it make a difference? Journal of Visual Impairment and Blindness, 95(4), 197-212.

Szarkowska, A. (2010). Accessibility to the media by hearing impaired audiences in Poland: Problems, paradoxes, perspectives. Approaches to Translation Studies, 32, 139-158.

Szarkowska, A., \& Laskowska, M. (2015). Poland - a voice-over country no more? A report on an online survey on subtitling preferences among Polish hearing and hearing-impaired viewers. In Bogucki, Ł. \& Deckert, M. (Eds.), Accessing Audiovisual Translation (pp. 179-197). Bern: Peter Lang.

Taylor, C. J., \& Mauro, G. (2012). The Pear Tree project: A geographico-statistical and linguistic analysis. Perspectives, 20(1), 25-41.

Toussaint, A.D. (Producer), \& Labaki, N. (Director). (2007). Caramel [Motion picture]. Lebanon: Sunnyland Films.

Vanderplank, R. (2016). Captioned media in foreign language learning: Subtitles for the deaf and hard-of-hearing as tools for language learning. London: Palgrave Macmillan.

Wissmath, B., Weibel, D., \& Groner, R. (2009). Dubbing or subtitling? Effects on spatial presence, transportation, flow, and enjoyment. Journal of Media Psychology, 21, 114-125. 